\author{
Journal of Research of the National Bureau of Standards \\ Volume 90, Number 6, November-December 1985
}

\title{
Polymers and Random Walks- Renormalization Group Description and Comparison With Experiment
}

\author{
Karl F. Freed \\ The University of Chicago, Chicago, IL 60637
}

\begin{abstract}
Accepted: July 1, 1985
Although real polymers involve the sequential addition of monomers having fixed bond lengths, fixed bond angles and some freedom of rotation about single bond, the properties of polymers over large length scales can be modeled by treating the polymer configuration as that of a random walk formed by the monomer units. Serious complications arise in the theoretical description of these polymers because of excluded volume constraints which prohibit different monomers from occupying the same position in space. This polymer excluded volume problem has been modeled in terms of a simple continuous random walk with short range repulsive interactions. The expansion of polymer properties in this repulsive interaction can readily be shown by dimensional analysis to involve an expansion in a large parameter, in the limit of long polymers. The renormalization group method is utilized as a systematic means for resuming this divergent perturbation expansion. The theory proceeds by analytically continuing the treatment to continuous range of spatial dimensionalities to expose and regularize the singularities in the analytically continued theory. The renormalization group approach is described fron a heuristic physical standpoint and extensive comparisons are provided to show how it quantitatively reproduces vast amounts of dilute solution polymer properties with no adjustable parameters.
\end{abstract}

Key words: experinent comparison; modeling; monomer units; polymer properties; random walk; repulsive interaction.

The study of the configuration statistics of polymers in dilute solutions presents problems of interest to analytical chemists, chemical physicists, engineers and applied mathematicians. Roughly half of the American chemical industry is involved with polymers, and analytical chemists are concerned with the characterization of their properties. Dilute solution physical properties are used to determine the molecular weight and general shape and architecture of the polymer. Hence, the availability of a quantitative theoretical description of the dependence of dilute solution physical properties on solvent characteristics, molecular weight, temperature, and branching is an important aid in characterizing and understanding the properties of polymers.

About the Author, Paper: Karl F. Freed is with the University of Chicago's James Franck Institute and Department of Chemistry. The work he describes was sponsored in part by the National Science Foundation.
The interest of polymers to applied mathematicians lies in their mathematical description in terms of interacting random walks.[1] $]^{1}$ This mathematical representation of a polymer chain can be motivated by visualizing the polymer as a sequence of bonded monomer units. Each new monomer, added to the chain, is attached by a chemical bond of fixed length, generally having fixed bond angles with respect to the previous bond. However, there is considerable freedom of rotation about the individual bonds thereby generating a large number of configurations for this idealized random walk polymer model [2]. The individual bond angle rotations represent random variables describing the chain configurations, so that when the number of these random variables, corresponding to the possible bond-vectors, gets large enough, the central limit theorem requires that the probability distribution for a vector between the ends of the chain must tend to a limiting Gaussian [1,2]. The random walk

\footnotetext{
${ }^{1}$ Figures in brackets indicate literature references.
} 
configurations of an idealized polymer permit two different segments of the polymer to occupy the same position in space, something which is not possible for real polymer molecules. Hence, real polymers are described in terms of interacting random walks with an excluded volume interaction prohibiting the multiple occupancy of monomers at the same place in space.

Experimental methods of polymer characterization include light scattering, osmometry, sedimentation, viscometry, etc. When the polymer concentration $c$ approaches zero; osmometry provides the determination of the molecular weight $M$ of the polymer, while small angle light scattering yields the polymers' radius of gyration $R_{\mathrm{G}}$. The limiting slope of the light scattering intensity as a function of concentration in the zero angle limit provides the polymer second virial coefficient $A_{2}$ which measures the effective volume that a polymer excludes to other chains. The translational diffusion coefficient $D$ is written for $c \rightarrow 0$ using Stokes' and Einstein's laws in terms of hydrodynamic radius $R_{\mathrm{H}}$ by $D=k T / 6 \pi \eta_{0} R_{\mathrm{H}}$ where $T$ is the absolute temperature, $k$ is Boltzmann's constant and $\eta_{0}$ is the solvent viscosity. If $\eta$ designates the viscosity of the polymer solution, the intrinsic viscosity $[\eta]=\operatorname{litm}_{c \rightarrow 0}\left(\eta-\eta_{0}\right) / c \eta_{0}$ gives another measure of the volume occupied by a single polymer chain.

All of these large scale observables for polymers provide different measures of the overall size and shape of the polymer. These properties are often found to vary with the polymer molecular weight in the form of a power law $K M^{a}$ where the proportionality factors $K$ and the exponents $a$ depend on the polymer, solvent, and temperature as well being slowly varying functions of $M$. It is the goal of a comprehensive quantitative theory of polymers [3] in dilute solution to explain the variation of $K$ and $a$ with the polymer-solvent system and the temperature over the full experimentally accessible range.

Because large scale polymer properties like $R_{\mathrm{G}}, A_{2}, D$ and $(\eta)$ are measures of large scale or long wave length polymer properties, the theoretical description of these polymer properties does not require a detailed treatment of the short range microscopic details of the polymer such as the specific bond lengths, bond angles and hindered rotation potentials. Rather, it suffices to employ apparently simple models to capture the essential large length scale characteristics of long chain molecules [3-7].

The above noted popular random flight model of polymers treats the chain as having a set of effective monomer units sequentially number $0,1, \ldots$, at the spatial positions $\mathbf{r}_{0}, \mathbf{r}_{1}, \ldots, \mathbf{r}_{n}$. Because the properties of interest involve large distance scales and implicitly large $n$, the central limit theorem allows us to take the individual bonds to have an effective Gaussian length distribution $[1,2]$ with an rms value of $l$. The excluded volume interaction is modeled by introducing a short range repulsive contribution to the energy when a pair of segments occupies the same position in space. This model then describes the dimensionless (free) energy associated with a chain configuration $\left\{\mathbf{r}_{k}\right\}$ of the form [1-4]

$$
\begin{aligned}
H / k T= & \left(d / 2 l^{2}\right) \sum_{j=1}^{n}\left|\mathbf{r}_{j}-\mathbf{r}_{j-1}\right|^{2} \\
& +\left(\beta_{0^{\prime}} 2\right) \sum_{i \neq j=0}^{n} \delta\left(\mathbf{r}_{i}-\mathbf{r}_{j}\right)
\end{aligned}
$$

where $\beta_{0}$ is the volume excluded by an effective segment due to the presence of another. The spatial dimensionality is $d$, and it is convenient to consider the description of polymers as a function of the dimensionality of space where normal solutions involve $d=3$ while the case $d=2$ is associated with polymers at a surface or an interface.

The probability distribution function for the chain configuration $\left\{\mathbf{r}_{k}\right\}$ is governed by the Boltzmann factor $\exp (-H /$ $k T$ ). Each of the monomer units in (1) describes the center of mass position of a collection of several actual monomers in the real polymer. This coarse graining is permissible because we are interested in long wavelength polymer properties. The first term on the right hand side in (1), therefore, represents the entropy of the polymer chain configuration associated with the many internal degrees of freedom in these coarse grain effective units [1-3]. Retention of only this term yields the simple Gaussian chain model of polymers at the theta temperature, a simple model for which the long wavelength polymer properties are easily evaluated. The entropic or elastic energy accounts in the model for the connectivity of the polymer chain.

The second term on the right hand side of (1) contains a pairwise sum over all the effective monomers and thereby converts the simple Gaussian chain model into a true manybody problem. The complexity of the treatment of excluded volume is readily seen by expanding $\exp (-H i k T)$ of $(\mathbf{I})$ in powers of $\beta_{0}$ and evaluating polymer properties as a formal power series in $\beta_{0}$. This excluded volume perturbation theory is readily shown [2-7] to be an expansion in the dimensionless quantity $\beta_{0} l^{-\mathrm{d}} n^{\epsilon / 2}$ with $\epsilon=4-\mathrm{d}$. Hence, for high molecular weight polymers where $n$ is large, the expansion is in a large parameter as long as $\beta_{0} l^{-\mathrm{d}}<n^{-\epsilon / 2}$ and $\mathrm{d}<4$. Consequently, the perturbation theory alone is of little use except very near the theta temperature where the empirical $\beta_{0}$ vanishes. The power law dependence of polymer properties on the molecular weight also indicates the difficulty of using these perturbation expansions in powers of $\beta_{0}$ since it is hard to see how a few terms in such an expansion, in a large parameter, can simply be resumed in order to provide the empirically observed nonanalytic power law dependence with fractional and often continuously varying exponents as a function of temperature. 
Renormalization group methods are designed specifically to effect the resummation of such asymptotic expansions in a large expansion parameter [3-10]. The theories work by analytically continuing the mathematical description to continuous dimensionality $\mathrm{d}$ in order to exhibit the singularities of the perturbation theory as a function of dimensionality [4-10]. As in analytic function theory, the singularities govern the dominant properties of the functional dependence on excluded volume and chain length and thereby enable resummation of the asymptotic perturbation expansion.

The process begins by noting that the perturbation expansion becomes a controllable one by expanding in powers of $\beta_{0}$ and of $\epsilon$. This $\epsilon$ expansion method yields for instance

$$
n^{\epsilon / 2}=1+(\epsilon / 2) \ln (n)+\left(\epsilon^{2} / 8\right) \ln ^{2}(n)+\ldots,
$$

so that when $n$ is large, a small $\epsilon$ can be chosen such that $\epsilon \ln (n)$ is a small expansion parameter. This procedure makes the perturbation expansion a mathematically well behaved method of computation, but it remains to show how perturbation expansions can be usefully applied to real $d=3$ or $\mathrm{d}=2$ polymers. The renormalization group method accomplishes the analytic continuation to $\mathrm{d}=3$ by focusing on the nature of the singularities of the perturbation expansion in powers of excluded volume for large scale polymer properties. The condition that the observable properties of polymers mathematically exist for $\mathrm{d}=4$ leads to a resummation approach which is embodied in the renormalization group equation that summarizes the general analytic dependence of large length scale polymer properties on the molecular weight and excluded volume interaction.

This renormalization group equation has implicit within it a coarse graining length scale $L$ that plays several important roles. First, $L$ is a phenomenological parameter which is used to average out short length scale properties of the theoretical model which are irrelevant in the description of large length scale polymer properties. Comparison with experiment shows $L$ to characterize a correlation range along the chain for excluded volume interactions [4]. Roughly speaking, a portion of the chain of length $L$ interacts with the remainder of the polymer as if this portion were effectively a hard sphere. When the excluded volume is weak, $L$ is comparable to the size of the polymer, so there is effectively no excluded volume interaction. At the other extreme of strong excluded volume, $L$ approaches an asymptotic limit [11] which can be taken as a useful empirical definition of the step length $l$.

The theoretical analysis uses a continuum limit of the energy (1) in which the Gaussian chain model alone would lead to Wiener integrals [1] and where the excluded volume term must be appended with a short distance cut off to remove counting of self-excluded volume interactions [3-9]. The double perturbation expansions in $\beta_{0}$ and $\epsilon$ are fairly straightforward albeit extremely tedious for the polymer properties of interest. The renormalized theory involves polymer properties on a coarse grain length scale $L$ in a region far from that for which perturbation expansions are valid. The physical properties are described in terms of the renormalized excluded volume $\beta$ and renormalized chain lengths $N$ replacing $\beta_{0}$ and the chain length $n l$ of the perturbation theory which is strictly valid only for $\beta_{0}$ very small. The details of the renormalization group method are too lengthy [3-10] to discuss here, so we turn to a description of some of the major results of the theory.

For instance, consider properties $Q$ for linear, ring, star and comb polymers like the radius of gyration or the hydrodynamic radius, etc., that naively scale as the $p$ th power of the polymer radius. The Gaussian chain value for this property is written as $Q_{0}=G_{Q}<S^{2}>_{0}^{\mathrm{p} / 2}$ with $\left\langle S^{2}>_{0}=\mathrm{N} l / 6\right.$ the Gaussian chain square radius of gyration $R_{\mathrm{G}}^{2}$. The property $Q_{0}$ is assumed to be known since it is relatively easily evaluated using the Gaussian chain model. An approximation to the second order renormalization group calculation in $\mathrm{d}=3$ yields $[7,11]$

$Q=\left\{\begin{array}{l}Q_{0}(1+32 \ddot{z} / 3)^{p / 8}\left[1+a_{Q}(32 \bar{z} / 3) /(1+32 \bar{z} / 3)\right], \bar{z}<0.75 \\ Q_{0}(6.441 \bar{z})^{p / 2 v-1)}\left(1+a_{Q}\right), \bar{z}>0.75,\end{array}\right.$

where $\nu=0.592$ to order $\epsilon^{2}$ and $a_{Q}$ is a pure number that depends only on the property $Q$ and emerges only from a first order calculation in $\epsilon$. The variable $\bar{z}$ is an empirical parameter which is often observed to depend on molecular weight and temperature in the form $\bar{z}=A M^{1 / 2}[1-(\theta / T)]$ with $A$ a polymer and solvent dependent quantity and $\theta$ the theta temperature where $A_{2}$ vanishes.

Perturbation expansions in excluded volume are effectively expansions in a parameter like $\bar{z}$. Expansion of (3) in $\bar{z}$ shows the expansion coefficients to be growing rapidly with the power of $\bar{z}$. Hence, eq (3) represents the results of a rather sophisticated resummation of the perturbation expansion in powers of $\bar{z}$ based in fact on only the first two terms in the series and an analysis of the singularities of this perturbation expansion as a function of the dimensionality of space. A slightly different form of the prediction emerges for quantities like second virial coefficient which vanish at the theta point, and the reader is referred to our previous papers for these simple analytic formulas $[7,11]$.

Figure 1 provides an example of the comparison between theory and experiment [12] for the interpenetration function $\psi(\bar{z})$ defined by

$$
A_{2}=\left(4 \pi<S^{2}>\right)^{3 / 2}\left(N_{A} / 2 M^{2}\right) \psi(\bar{z})
$$

as a function of the radius of gyration expansion factor defined by

$$
\alpha_{S^{2}}^{2}=<S^{2}>/<S^{2}>_{0}
$$




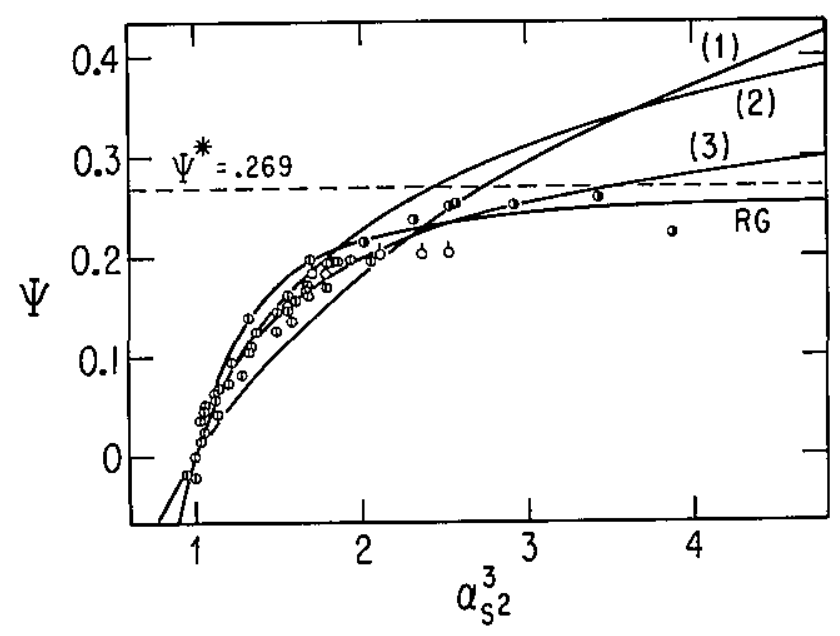

Figure 1-Comparison of theories [2] and experiment for variation of $\psi$ with $\alpha_{S^{2}}^{3}$ [3]. The figure is reproduced from Yamakawa [2] with the parameter free RG predictions added. Data points from Norisuye et al. [14] are for polychloroprene (PC) in $\mathrm{CCl}_{4}$ at $25^{\circ} \mathrm{C}, O$ for $\mathrm{PC}$ in $n$-butyl acetate at $25^{\circ} \mathrm{C}$, and $\bigcirc$ for $\mathrm{PC}$ in transdecalin at various temperatures. Similar data [15] and agreement with theory is available for polyisobutylene, polystryrene and poly-p -methylstyrene in various solvents. The curves (1)-(3) represent older theories as reviewed by Yamakawa [2].

The renormalization group prediction is given by the solid line marked RG, whereas the other lines represent older theories [2] which lack systematic mathematical guidance and therefore which are unable to predict the physically obvious fact that $\psi$ must lead to a universal value in the limit of large chain expansion or equivalently large excluded volume interaction $\bar{z}$.

An important feature of figure 1 is that the theoretical curve is obtained with absolutely no adjustable parameters, and that it is also derived using purely analytical methods. Hence, its derivation is in the true spirit of analytical chemistry, using the methods of mathematical analysis to provide quantitative descriptions of the properties of chemical systems. This type of work merges the disciplines of analytical chemistry and applied mathematics and is therefore at the heart of the goals of chemometrics.

Renormalization group calculations have been performed for a wide range of polymer properties [12] in dilute solutions for linear, ring, star, and block copolymers. The agreement between theory and experiment is generally as good or better than that presented in figure 1 . The situation is somewhat more complicated for the dynamical properties $R_{\mathrm{H}}$ and $[\eta]$ where our theoretical calculations show that the effective exponents $a$ in good solvents often depend on an additional parameter, called the draining parameter [13]. We believe that the success of this renormalization group description of the excluded volume dependence of dilute solution properties will enable us to describe polymer properties in a variety of mathematically more complicated and physically very interesting situations such as the properties of polymers in interaction with a surface or interface, the properties of polyelectrolyte solutions where polymers have a distribution of charges and there are small counter ions in solution, and the properties of polymer mixtures in solution.

I am grateful to Jack Douglas for comments on the manuscript.

\section{References}

[I] A more mathematically oriented introduction to polymers as interacting random walks is given in K.F. Freed, Ann. Prob. 9, 537 (1981), a paper which predates our current work with the more powerful renormalization group methods.

[2] See, for instance, H. Yamakawa, Modern Theory of Polymer Solutions (Harper and Row, New York, 1971) and references therein.

[3] Freed, K.F., Accts. Chem. Res. 18, 38 (1985).

[4] Oono, Y., T. Ohta and K.F. Freed, J. Chem. Phys. 74, 6458 (1971).

[5] Oono, Y., and K.F. Freed, J. Phys. A 15, 1931 (1982).

[6] Kholodenko A.L., and K.F. Freed, J. Chem. Phys. 78, 7390 (1983).

[7] Douglas, J.F., and K.F. Freed, Macromolecules 17, 1854 (1984).

[8] Amit, D.J., Field Theory. The Renormalization Group and Critical Phenomena (McGraw-Hill, New York, 1978).

[9] Ramond, Field Theory, A Modern Primer (Benjamin/Cummings. Reading, Mass., 1981).

[10] Freed, K.F., Renormalization Group Theory of Macromolecules (Wiley, to be published).

[11] Douglas, J.F., and K.F Freed, Macromolecules 15, 1800 (1983).

[12] Douglas, J.F., and K.F. Freed, Macromolecules 17, 2344 (1984); 18 , 201 (1985).

[13] Douglas, J.F., and K.F. Freed, Macromolecules 17, 2354 (1984).

[14] Norisuye, T., K. Kawahara, A. Teramoto and H. Fujita, J. Chem. Phys. 49, 4330 (1968); K. Kawahara, T. Norisuye and H. Fujita, J. Chem. Phys. 49, 4339 (1968).

[15] Berry, G.C., J. Chem. Phys. 44, 4550 (1966); G. Tanaka, S. Imai and H. Yamakawa, J. Chem. Phys. 52, 2639 (1970); T. Matsumoto, N. Nishioka and H. Fujita. J. Polym. Sci. 10, 23 (1972). 